\title{
Survey of the stability region of hypothetical habitable Trojan planets
}

\author{
R. Schwarz ${ }^{1,2}$, R. Dvorak ${ }^{1}$, Á. Süli ${ }^{2}$, and B. Érdi ${ }^{2}$ \\ ${ }^{1}$ Institute for Astronomy, University of Vienna, Türkenschanzstr. 17, 1180 Wien, Austria \\ e-mail: schwarz@astro.univie.ac.at \\ 2 Department of Astronomy, Eötvös University, Pázmány Péter sétány 1/A, Budapest 1117, Hungary
}

Received 1 June 2007 / Accepted 13 August 2007

ABSTRACT

\begin{abstract}
Aims. In this work we study the dynamical possibility in extrasolar planetary systems that a terrestrial planet can exist in 1:1 mean motion resonance with a Jovian-like planet. We compiled a catalogue of hypothetical habitable Trojan planets, to be able to make a stability forecast for further extrasolar planetary systems discovered in the future. When speaking of habitability we also took the influence of the spectral type of the central star into account.

Methods. We integrated some $10^{6}$ orbits of fictitious Trojans around the Lagrangian points for up to $10^{7}$ orbital periods of the primary bodies and checked the stability of the orbital elements and their chaoticity with the aid of the Lyapunov characteristic indicator and maximum eccentricity. The computations were carried out using the dynamical model of the elliptic, restricted three-body problem that consists of a central star, a gas giant moving in the habitable zone, and a hypothetical (massless) terrestrial planet.

Results. Our investigations have shown that 7 exoplanetary systems can harbour habitable Trojan planets with stable orbits (HD 93083, HD 17051, HD 28185, HD 27442, HD 188015, HD 99109, and HD 221287, which is a recently discovered system). The comparison of the investigated systems with our catalogue showed matching results, so that we can use the catalogue in practice.
\end{abstract}

Key words. celestial mechanics - stars: planetary systems - astrobiology

\section{Introduction}

Does life exist elsewhere in the universe and where can we find it? When astronomers look for life on planets in exoplanetary systems (EPS), they have to know the location of the so-called habitable zone (HZ), which is defined as a region around a star where a planet would receive enough radiation to maintain liquid water on its surface and to be able to build a stable atmosphere. The HZ is estimated via climate calculations, which can be used to describe the possible surface conditions on a terrestrial planet moving in this zone. Its distance and width depend on the spectral type and the age of the central star (see Kasting et al. 1993). But there are other severe constraints concerning habitability on any planet moving in such a favourable zone. Besides the requirement for the mass of the planet $\left(<8 M_{\oplus}^{1}\right.$, Lammer et al. 2005), the terrestrial-like planet needs to have a crust that allows plate tectonics and is also necessary for building an atmosphere and later forming a biosphere.

Out of more than 220 planets in 180 EPSs, we only have observational evidence of planets ranging from $5 M_{\oplus}$ (Gliese $581 \mathrm{c}$, see Udry et al. 2007) up to several Jupiter-masses. To answer the question of life in the universe other than on our Earth, we have to search for different possible configurations $(\mathrm{C} 1-\mathrm{C} 3)$ of dynamically, stable habitable planets with respect to the orbit of a Jovian planet or gas giant (GG):

- C1: when the GG is very close to the star, a habitable planet could exist with a stable orbit in the HZ, which is located outside the GGs orbit.

\footnotetext{
$11 M_{\oplus}$ is equivalent to 1 Earth-mass.
}

- C2: when the GG moves far away from the central star (like Jupiter), it allows additional planets to move on stable orbits closer to the star inside the HZ.

- C3: when the GG itself moves in the HZ, a habitable satellite (like e.g. Titan in the system of Saturn) could have a stable orbit, or a Trojan-like habitable planet may move in a stable orbit around the Lagrangian equilibrium points $L_{4}$ or $L_{5}$.

In our work we concentrate on investigating Trojan-like habitable planets ${ }^{2}$ (C3 type). In Table 1 we show a list of possible EPSs, where the GG's orbit has low-to-moderate eccentricities $(e<0.3)$ and moves partly or fully inside the HZ. Column 3 represents the mass of the central star, Col. 4 the mass of the GG, and Col. 7 the limits of the HZ (see Kasting et al. 1993). The list is sorted by increasing semimajor axis of the GG and shows the size of the stable area for a calculation time of $10^{7}$ periods. Dynamical studies exist of fictitious Trojan planets in ESPs like Schwarz et al. (2007a), Schwarz et al. (2005), Schwarz (2005) and Dvorak et al. (2004). All of them aim at finding the extension of the stable regions around the equilateral equilibrium points $L_{4}$ and/or $L_{5}$. Several authors have done similar studies for the Trojan asteroids in our Solar system e.g.: Marzari \& Scholl (1998), Morbidelli et al. (2005). Beaugé et al. (2007) have also investigated the possible formation and evolution of coorbital terrestrial planets in EPSs. Most of the studies of Trojan planets have been undertaken without taking their masses into account,

\footnotetext{
2 In the solar system, Trojans are two groups of asteroids moving close to the equilibrium points (Lagrangian points) $L_{4}$ and $L_{5}$ in 1:1 mean motion resonance with Jupiter. That means that the Jupiter's Trojans are moving either close to $60^{\circ}$ ahead or $60^{\circ}$ behind Jupiter with nearly the same semimajor axis as the planet.
} 
Table 1. List of all EPSs with one giant planet in the HZ.

\begin{tabular}{llllllll}
\hline \hline Name & $\begin{array}{l}\text { Stable area } \\
{\left[\mathrm{AU}^{2}\right] \text { for }} \\
10^{7} \text { periods }\end{array}$ & $\begin{array}{l}\text { Mass } \\
{\left[M_{\text {sol }}\right]}\end{array}$ & $\begin{array}{l}\text { Mass } \\
{\left[M_{\text {jup }}\right]}\end{array}$ & $\begin{array}{l}a \\
{[\mathrm{AU}]}\end{array}$ & ecc & $\begin{array}{l}\mathrm{HZ} \\
{[\mathrm{AU}]}\end{array}$ & $\begin{array}{l}\text { Partly } \\
\text { in HZ } \\
{[\%]}\end{array}$ \\
\hline HD 101930 & 0.0637 & 0.74 & 0.30 & 0.30 & 0.11 & $0.30-0.64$ & 53 \\
HD 93083 & 0.0781 & 0.70 & 0.37 & 0.48 & 0.14 & $0.28-0.60$ & 100 \\
HD 134987 & 0.0246 & 1.05 & 1.58 & 0.78 & 0.24 & $0.75-1.40$ & 58 \\
HD 17051 & 0.0321 & 1.03 & 1.94 & 0.91 & 0.24 & $0.70-1.30$ & 100 \\
HD 28185 & 0.0587 & 0.99 & 5.70 & 1.03 & 0.07 & $0.70-1.30$ & 100 \\
HD 99109 & 0.3254 & 0.93 & 0.50 & 1.11 & 0.09 & $0.65-1.25$ & 100 \\
HD 27442 & 0.3967 & 1.20 & 1.28 & 1.18 & 0.07 & $0.93-1.80$ & 100 \\
HD 188015 & 0.1148 & 1.08 & 1.26 & 1.19 & 0.15 & $0.70-1.60$ & 100 \\
HD 1 14783 & 0.2566 & 0.92 & 0.99 & 1.20 & 0.10 & $0.65-1.25$ & 50 \\
HD 221287 & 0.1857 & 1.25 & 3.09 & 1.25 & 0.08 & $1.10-2.30$ & 100 \\
HD 20367 & 0.0576 & 1.05 & 1.07 & 1.25 & 0.23 & $0.75-1.40$ & 76 \\
HD 23079 & 0.2099 & 1.10 & 2.61 & 1.65 & 0.10 & $0.85-1.60$ & 35 \\
\hline
\end{tabular}

because they are quite small compared to a Jupiter-like planet (Menou \& Tabachnik 2003; Dvorak et al. 2004). Érdi \& Sándor (2005) determined the stability region around $L_{4}$ in the elliptic, restricted three-body problem (ER3BP), depending on the mass ratio of the primary bodies and their eccentricities. The influence of secondary resonances between the frequencies of librational motions around $L_{4}$, visible in a minimum of the size of the stable region, were investigated in the circular case by Tuckness (1995) and for the ER3BP by Erdi et al. (2007b). There are other investigations in which the authors (e.g. Nauenberg 2002; Laughlin \& Chambers 2002) also study massive Trojan planets. Recently, Érdi et al. (2007a) used the results of short time computations of the relative Lyapunov Indicator (RLI) for compiling a catalogue showing the stable regions where they also took massive Trojans into account in their computations.

One aim of our investigation was to make a dynamical study of all real EPSs that are fulfilling the requirements for hosting stable Trojan planets (see Table 1). The other task using results of long-term integration is to compile a new catalogue where the extension of the stable regions around the equilibrium points depending on the mass ratio of the primaries and the eccentricity of the orbits of the primaries are computed. All these studies were undertaken in the framework of the ER3BP with a cloud of massless Trojans set close to the Lagrangian point $L_{4}$.

\section{Methods of investigation}

Because of the smallness of an Earth-like planet, one can use the ER3BP as a dynamical model for an EPS with one giant planet. One can neglect the mass of the Trojan planets since the stability character of the equilateral Lagrangian points and the extension of the stability zone does not change significantly with a small third mass, which is shown in Marchal (1990) and Schwarz et al. (2005).

The goal of this investigation is to establish the size of the stable regions around the equilateral Lagrangian points in the ER3BP depending on the two parameters of the problem: the mass ratio of the primaries and the eccentricity of their respective orbits. This has been undertaken with the aid of numerical simulations of millions of orbits of fictitious Trojans in the vicinity of $L_{4}$. The case of $L_{5}$ is exactly symmetric in the chosen model of the ER3BP; consequently, when we mention $L_{4}$ during this article we also mean $L_{5}$. This symmetry does NOT exist in a system with an additional perturbing planet.

From analytical works in the circular restricted three-body problem, we have some estimates of these stability zones in our Solar System. These results were derived by applying the Nekhoroshev theorem, which allows establishing an effective time for which a massless body stays in a certain region around one of the equilateral equilibrium points; see e.g., Giorgilli \& Skokos (1997), Skokos \& Dokoumetzidis (2001) and Efthymiopoulos \& Sándor (2005). With this sophisticated method, it is possible to say that about $30 \%$ of the Jupiter's Trojans will stay close to the Lagrangian points for time scales longer than the age of our Solar system. (Note that these estimations were made by neglecting Jupiter's orbital eccentricity and the influence of the other planets.) The only practical way to establish stability limits for the stable regions around the triangular equilibrium points of extrasolar planetary systems is with the aid of extensive numerical integrations of test planets. We limit ourselves to an initial eccentricity of the GG smaller than 0.3 , because our previous investigations show (Dvorak \& Schwarz 2005; Schwarz et al. 2007b) that Trojan planets below this limit can have stable orbits for long times. This is also important for the question of habitability. In general the orbital habitability (which means the test particle stays $100 \%$ in the $\mathrm{HZ}$ during the integration time) of a possible Trojan planet depends on 3 factors: the eccentricity, the mass ratio, and the extension of the HZ that depends on the spectral type of the star. If we set the eccentricity of a planet in the $\mathrm{HZ}$ to $e_{\mathrm{GG}}<0.3$, then the planet could be outside the $\mathrm{HZ}$ at some points on its orbital path. A former work of Schwarz (2007) shows that above this limit the fictitious Trojan planet cannot stay $100 \%$ in the HZ for any mass ratio of the primaries.

\subsection{Numerical methods}

We have written a numerical code based on a Bulirsch-Stoer integrator where we do not have to integrate the orbits of the primaries. The advantage is to use rotating, pulsating coordinates, where there is no need to find a solution for the motion of the primaries. In addition, one can integrate many orbits of the massless fictitious bodies simultaneously. To verify some results we also used the Lie-method. More details about this method are given in Hanslmeier \& Dvorak (1984) and Lichtenegger (1984). For analysing the stability, we checked the maximum eccentricity of the Trojan planets during its dynamical evolution (maximum eccentricity, $e_{\max }$ ), which was already used extensively by us and does not need any additional computations. This method turned out to be very useful and immediately tells the stability of an orbit. The stability criterion for a Trojan was that the eccentricity should not exceed an eccentricity of 0.5 . All test computations 


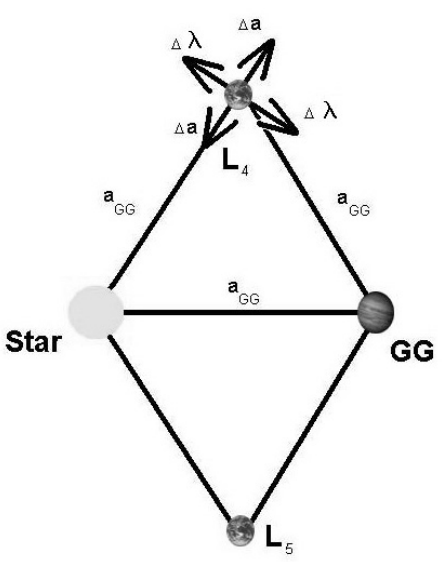

Fig. 1. The figure shows how the orbital elements of the Trojan planets (the semimajor axis $\Delta a$ and the synodic longitude $\Delta \lambda$ ) were changed. We have to remark that this figure is only schematic, because it presents the circular case, in the elliptic case the distance between the star and $L_{4}$ is changing with the period of the primaries.

have shown that setting $e_{\max }=0.5$ for the fictitious body as the stability limit is very effective because continuing the computations made all orbits unstable (e.g. crossed the connecting line of the primaries). As a complementary tool, we computed also the Lyapunov characteristic indicator (LCI), a well-known chaos indicator. The LCI is the finite time approximation of the largest Lyapunov exponent (LCE), which is decribed in detail in e.g. Froeschlé (1984) and Lohinger et al. (1993a).

\subsection{Determination of the stable area}

An important point is the choice of the grid for the initial conditions around $L_{4}$ and the time interval of the integration for one set of the parameters of the system. In Fig. 1 we show how we have chosen the initial conditions around the Lagrangian point $L_{4}$ : in one direction, along the connecting line of the star and the Trojan planet (see $a_{\mathrm{GG}}$ in Fig. 1), we changed the normalised semimajor axis $a$ of the Trojan planet, and in the other direction we changed the synodic longitude $\lambda$. The synodic longitude of the fictitious planet is the difference between the mean orbital longitudes of the fictitious planet and the giant planet. It is evident that a fine scaling of the grid would be desirable (see Fig. 3). Although we use supercomputers, the two-parameter study would make the necessary CPU-time for the integration too long. An important choice concerns the integration time. Only short-term integrations were used in previous results using chaos indicators (up to $10^{3}$ periods). Chaos indicators can be used to distinguish between regular and chaotic motion, but a direct test of the orbits (e.g. eccentricity) reveals the character of its motion in a quantitative way after long term integrations. We therefore have taken our simulations for timescales up to $10^{7}$ periods of the primaries. In general we used the Bulirsch-Stoer-method and calculated the stability maps by varying the normalised semimajor axis and the synodic longitude.

It turned out that the stability region has a form that can be approximated by an ellipse (compare Fig. 3). Cuts of the stable region were made in two directions (with a gridsize given below). First we fixed $\lambda$ at $60^{\circ}$ and computed the orbits of the fictitious objects in the direction of $a$ with a special grid-size $(\delta a)$. The second cuts were made in the opposite direction ( $a$ was fixed at $1[\mathrm{AU}]$ and $\lambda$ was varied); these cuts are visualised in Fig. 2.

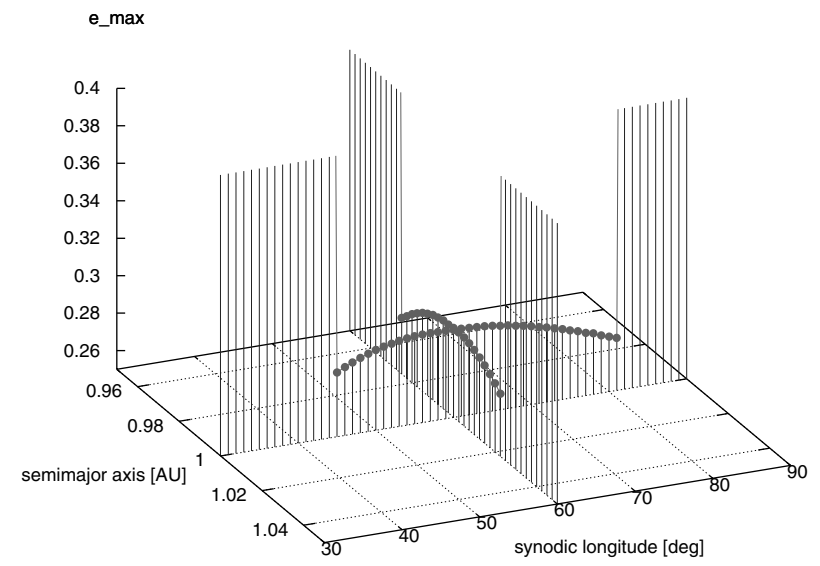

Fig. 2. Cuts of the stable region in the directions of $a$ and $\lambda$, for the catalogue $(e=0.15$ and $\mu=0.002)$.

\section{Results of real systems with a GG in the $\mathrm{HZ}$}

First we investigated the extension of the stable regions of the 12 real systems ${ }^{3}$, given in Table 1 . Therefore we made test calculations for 2 different integration times $\left(10^{4}\right.$ and $10^{6}$ periods). For the grid of the stability-maps (see Fig. 1), we changed $0.9<a<1.1$ with a grid-size $\Delta a=0.0025$ and $0^{\circ}<\lambda<180^{\circ}$ with a grid-size $\Delta \lambda=1^{\circ}$. We want to point out that, for all stability-maps, we calculated the LCI and $e_{\max }$, but only we show the LCI. We integrated for $10^{4}$ periods (see Fig. 3 upper graph) and $10^{6}$ periods (see Fig. 3 lower graph). When we compare these two stability maps, we can conclude that the ring-like structure is smaller after $10^{6}$ periods and will maybe disappear after $10^{7}$ periods. That is why we later decided to calculate the catalogue up to $10^{7}$ periods. The results of the size of the stable region - approximated by an ellipse - are given in Table 1 .

Table 1 shows all extrasolar planetary systems where only one giant planet moves partly or fully in the HZ. The ER3BP was therefore taken as the dynamical model, where $L_{4}$ and $L_{5}$ are perfectly symmetric (the equations of motion are symmetric in time). We stress that this symmetry is "not" true when we check the regions around the equilateral equilibrium points in the Solar System. The paper by Holman \& Wisdom (1993) found that the two equilibrium points are symmetric for Jupiter, Saturn (although completely unstable after $10^{6}$ years), and Uranus. But for Neptune Trojans, the region around $L_{5}$ is shifted towards larger semimajor axes. Inclined Neptune Trojan orbits are much stabler than orbits in the plane of motion of Neptune, as is also known from the distribution of the real Jupiter Trojans.

The stability maps of the real systems show us that some of them could in fact have Earth-like satellites and/or Earth-like Trojans; see e.g. Dvorak et al. (2004), Érdi \& Sándor (2005), Schwarz et al. (2005). There are 7 systems where one GG is fully in the HZ; 5 (HD 93083, HD 17051, HD 28185, HD 27442, and HD 188015) were investigated in Schwarz (2007a) and one, HD 99109, in Schwarz et al. (2007). As in Schwarz (2007a), we also investigated the stable region of all systems by using the cuts for an integration-time up to $10^{7}$ periods. The results of the size of the stable region are shown in Table 1 for $10^{7}$ periods. The systems HD 27442, HD 99109, HD 23079, and HD 114783 have the largest stable regions.

\footnotetext{
3 That means: extrasolar planetary systems where one GG moves partly or fully in the HZ and that a possible earth-like Trojan planet can have a stable orbit.
} 

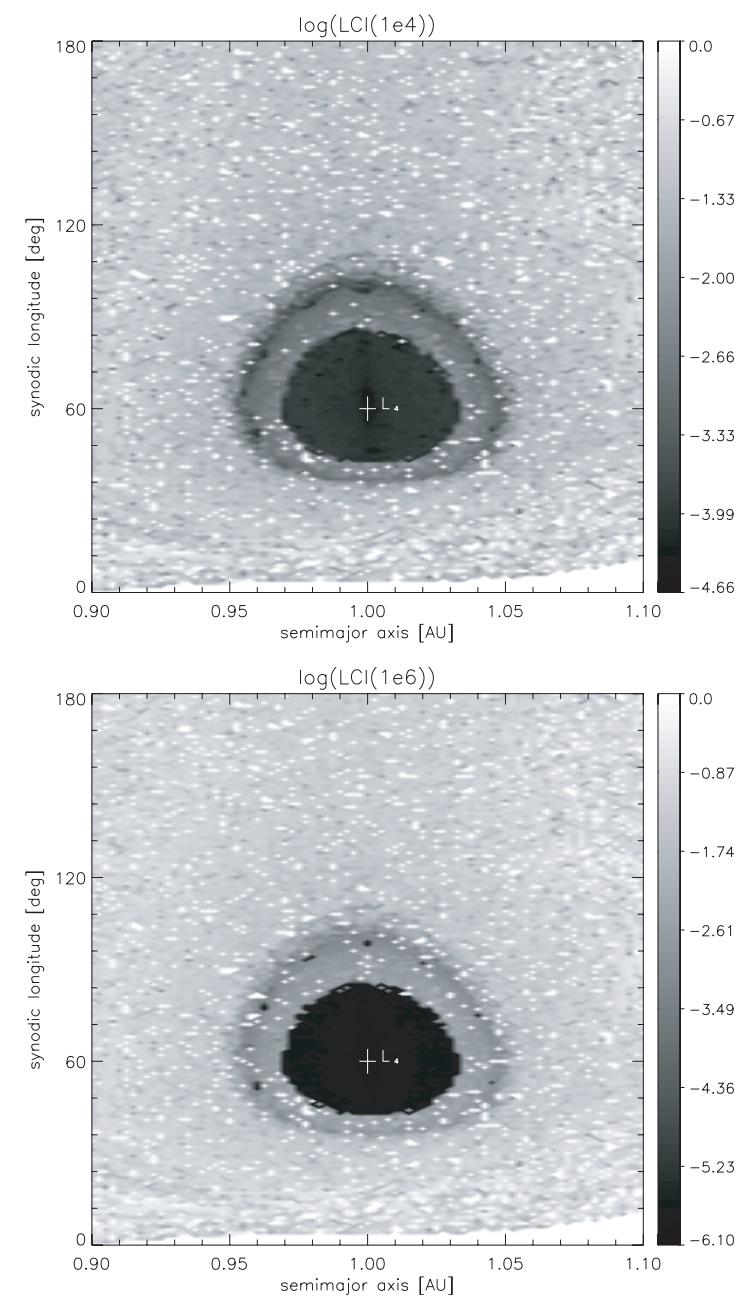

Fig. 3. Stability map of the system HD 221287 around the equilibrium point $L_{4}$ for $10^{4}$ periods (upper graph) and for $10^{6}$ periods (lower graph). We used the LCI to check the stability. The dark regions indicate stable orbits, and the light one chaotic orbits. On the $x$-axis, we plotted the initial semimajor axis and the initial synodic longitude of the Trojan planet on the $y$-axis.

\section{The catalogue}

A systematic investigation exist for the extension of the stability region around the equilibrium points for a wide range of the eccentricity and the mass ratio of the primaries by Lohinger \& Dvorak (1993). The nonlinear stability domain of Lagrange's celebrated 1772 solution to the three-body problem is obtained numerically as a function of the masses of the bodies and the common eccentricity of their Keplerian orbits. More recently, Érdi \& Sándor (2005) have determined via the computation of the RLI this region. The main reason for redoing the investigations was the extension to long-term integrations up to $10^{4}$ times longer than the formerly mentioned one, and we concentrate our investigations on a mass ratio up to $\mu=0.01$ and an eccentricity lower than 0.3 . This was because we are interested in possibly habitable Trojan planets. The third-body motion was studied in the ER3BP, because former investigations showed that a lower mass of the Trojan planet compared to the second primary does not change the stability picture very much, presented in Érdi et al. (2007a).

Again we used the cuts like the one in the Sect. 3 to determine the size of the stability regions. The grid of the stabilitymap was $0.9<a<1.1$ with $\Delta a=0.0025$ and $0^{\circ}<\lambda<180^{\circ}$
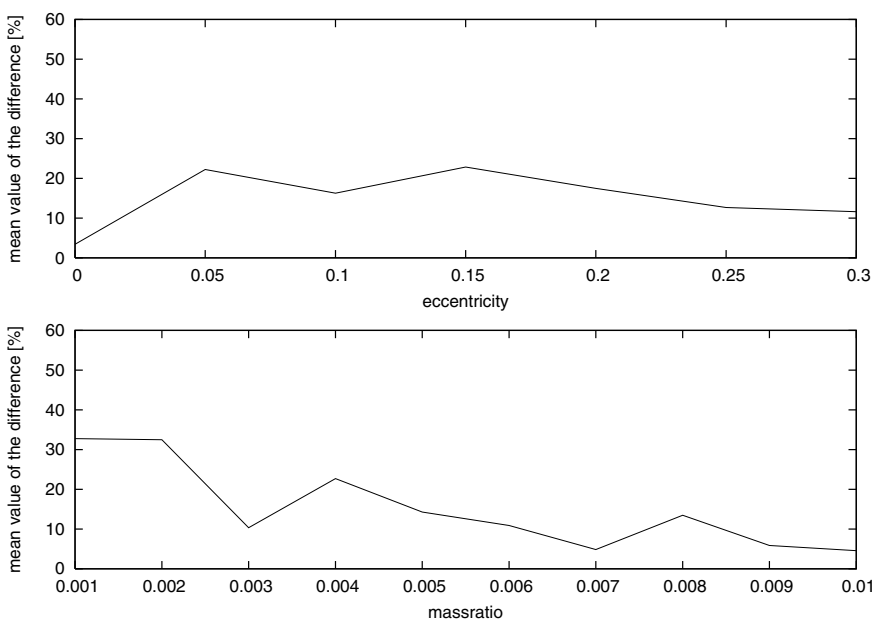

Fig. 4. These figures depict the mean value of the difference [\%] between the two integration-times of the $10^{4}$ and $10^{7}$ periods. The upper graph shows the mean value for the 7 initial eccentricities (of the 20 different mass ratios) and the lower one for the mean value for the 20 initial mass ratios (for 7 different eccentricities).

with $\Delta \lambda=1^{\circ 4}$. The cuts were made for the $10^{4}$ and $10^{7}$ periods and the limit of the eccentricity was set to 0.3 (grid-size $\Delta e=0.05$ ), the mass ratios were set to $\mu=0.01$ (grid-size $\mu=0.001$ ). We know that GG with a mass higher than 10 Jupiter (at $\mu=0.01$ ) masses are brown dwarfs. The limit between a brown dwarf and a GG is not clear, because it depends on their interior structure. In our definition we say that the mass ratio $\mu<0.01$ is the respective limit.

The next step was to test how accurate these calculations are for $10^{4}$ periods compared with $10^{7}$ periods. For this we calculated the difference in the size of the stable regions for all cuts up to $\mu=0.01$ for the two different time scales with $10^{4}$ and $10^{7}$ periods. Therefore we computed the mean value of each of the eccentricities (Fig. 4 upper graph) and for the different mass ratios. That means we calculate the mean value for each eccentricity ( 7 values), which we get from the 20 different mass ratios of the primaries than we made this contrary with each of the mass ratios (Fig. 4 lower graph). It is clear that the difference for the various eccentricities has a maximum at 0.05 and 0.15 and for the mass ratios at low values $\mu \leq 0.002$. We can summarise that the mean values are about $20 \%$ (see also Schwarz et al. 2007a). This is a first estimate for predictions of possible Trojan planets in newly discovered systems.

We emphasise that, for the estimates of the size of the stable regions, it is not relevant whether we approximate the area by an ellipse $(A=a b \pi)$ or, for example, by a deltoid $\left(A=\frac{e f}{2}\right)$. With this method it was possible to use a finer grid in the eccentricity and mass ratio to estimate the two axes of the ellipse and to compute the size of the stable area for the catalogue. Because of using normalized semimajor axis, we can easily compare different stability areas for different exoplanetary systems.

In Fig. 5 we present the size of the stable area (given in $\left.\left[\mathrm{AU}^{2}\right]\right)$. The grey-scale of the stable area goes from 0.05 to $0.5\left[\mathrm{AU}^{2}\right]$. To visualise how large the stable area around an extrasolar Lagrangian equilibrium point could be, we calculated the area inside the orbit of Mercury and got an area of $0.43\left[\mathrm{AU}^{2}\right]$. When we compare that with the grey-scale shown

4 The eccentricity of the Trojan was the same as for the GG, and $\omega$ of the Trojan was always set to $\omega_{\text {Trojan }}=\omega_{\mathrm{GG}}+60^{\circ}+$ the respective variation in $\omega$. 

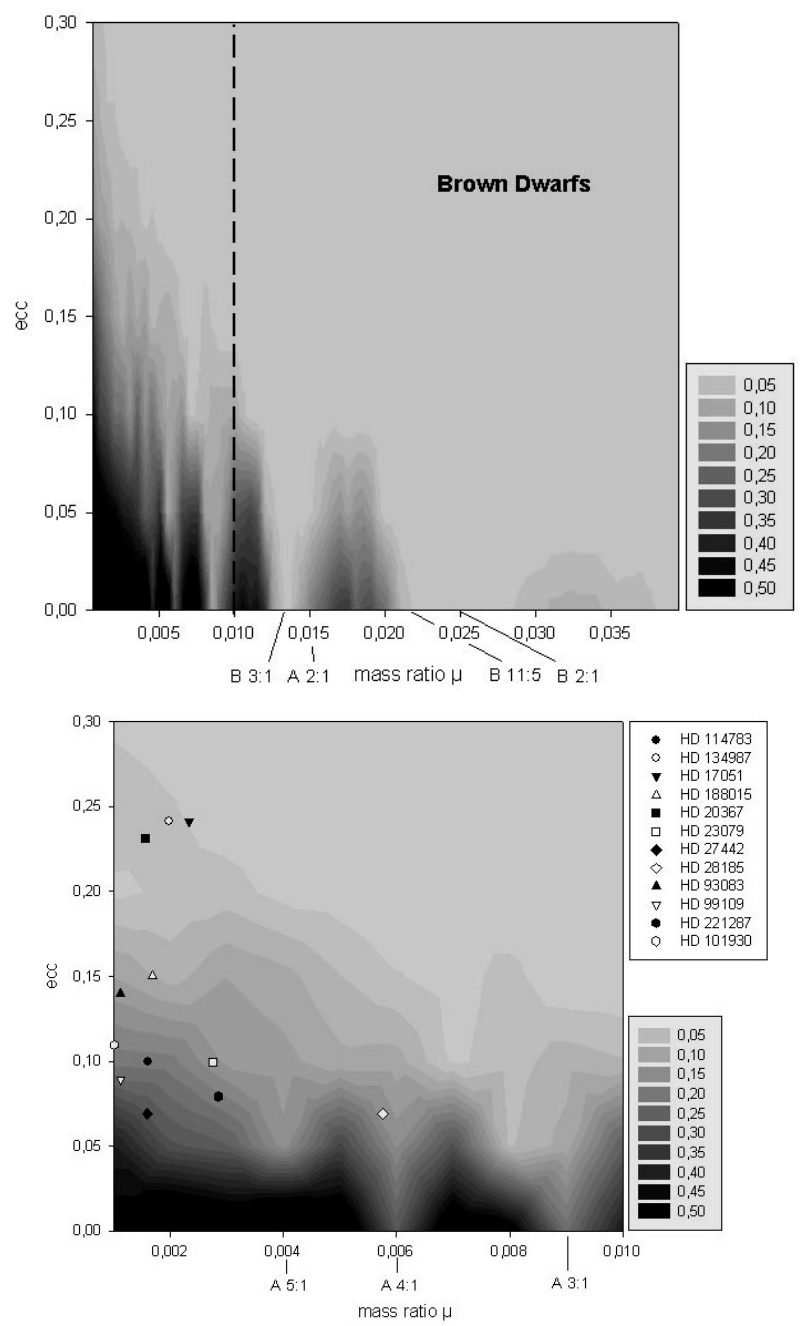

Fig. 5. The size of the stability regions given in $\left[\mathrm{AU}^{2}\right]-$ values between 0.05 and 0.5 - around an equilateral equilibrium point in the ER3BP. We varied the mass ratio in the $x$-direction and the eccentricity of the primaries' orbit in the $y$-direction. The values of the grey-scale present the size of the stable region (dark region indicates a large stable region and the light one a small). The upper graph shows the stable area for $10^{4}$ periods and the lower for an integration-time of $10^{7}$ periods and the upper one for higher mass ratios up to $\mu=0.04$.

in Fig. 5, we can conclude that the stable regions could reach higher values than the area of the orbit of Mercury (mainly for the circular case, but also for very low eccentricities).

The rough structure of the global picture coincides quite well with the work of Érdi \& Sándor (2005). More details can be seen in our Fig. 5 (upper graph), where we used a finer grid in mass ratio $\Delta \mu=0.0005$. The finger-like structures are separated by high-order resonances between the libration periods of the massless body. We marked the 2:1 and the 3:1 resonances, which are pointed out in the work of Érdi et al. (2007b). These resonances of the motion around the equilibrium points are determined by the two frequencies $n_{\mathrm{s}}$ and $n_{\mathrm{l}}$, which are the corresponding short and long period components of the libration and the mean motion $n$ (shown in Fig. 5). If we combine the frequencies $n_{\mathrm{s}}$ and $n_{\mathrm{l}}$ with that of the motion around $L_{4}$, we get four frequencies and linear combinations of them. That means that in the circular problem we have: $n_{\mathrm{s}}, n-n_{1}, n_{\mathrm{l}}$ and $n-$ $n_{\mathrm{s}}$. There are 6 possible types of resonances (more details in
Erdi et al. 2007b), and both are important for our investigation: the A type resonance $\left(n-n_{1}\right): n_{1}$ and the B type resonance $n_{\mathrm{s}}: n_{\mathrm{l}}$.

Because the mass parameter in ESPs is of interest between 1 and 10 Jupiter masses we zoomed in this region and extended the integrations to $10^{7}$ periods (Fig. 5 lower graph). The catalogue was normalised to $1 \mathrm{AU}$ (shown in Figs. 3 and 2), because the $\mathrm{HZ}$ close to $1 \mathrm{AU}$ for G-stars is in most cases. If the GG is not at $1 \mathrm{AU}$ distance to the central star, it is necessary to adapt the stable area. Thus we have to recalculate values of the stable area from the catalogue to that of the real system. This is only a simple scale transformation: the axes of the stable area $(a, \lambda)$ are scaled to the unit circle at $1 \mathrm{AU}$ and can be easily recalculated by dividing them by the semimajor axis of the real system.

The catalogue also shows the 12 real systems that were investigated. In the upper and lower graphs (Fig. 5), it is clear that the unstable regions also correspond to secondary resonances (see Érdi et al. 2007b), at the mass ratios $\mu=0.004$ (caused by A $5: 1$ ) $\mu=0.006$ (A 4:1), $\mu=0.009$ (A 3:1), $\mu=0.014$ (B 3:1), $\mu=0.015$ (A 2:1), $\mu=0.022$, and $\mu=0.01$ (B 2:1) between the libration periods of the Trojan planet. These are the resonances that perturb the orbits of the Trojan planets and change the size (visible as gaps in this figure) of the stable regions.

Tuckness (1995) also investigated the stability around $L_{4}$, but only the dynamical model of the circular restricted problem, by using the positional or velocity deviation of the test particles away from the equilibrium point point $L_{4}$. He also approximated the stability region by an ellipse. When we compared the work with that of Tuckness, we found agreement that the B 3:1 and the B 2:1 plays a major role in the determining the stability.

The results of both graphs present the following: the stable region is large close to the $e_{\text {primaries }}=0$ (the circular problem) and low mass ratios (light grey indicates no stable area, middle to dark-grey - large to small - stable area). The large bright region outside (higher eccentricity and higher mass ratio, small stable region) depicts more escaping orbits around the equilibrium points. In this figure we can see that, for higher eccentricities (up to 0.3 ) and lower mass ratios $(\mu \leq 0.005)$, Trojan planets can have stable orbits. This is important because all of the real systems are in this region.

\section{Habitable Trojan planets in the context of the spectral type of the central star}

The search for signs of life in EPSs concentrates on systems where the host star is located on the main sequence of the Hertzsprung-Russell diagram (HRD). Climate models estimate the width of the $\mathrm{HZ}$ around a host star depending on its mass, spectral type and age (Kasting et al. 1993). The habitability of a possible Earth-like planet will be limited by different effects of the host star, for example, the stellar lifetime of main sequence stars decreases strongly with the mass of the star (see Fig. 6).

Dole (1964) pointed out that planets in the HZ of M-type stars might be synchronously rotating as a result of tidal locking $^{5}$. Another problem with M-type stars is that they have significant flare activity that prevents a planet from having a long-time stable atmosphere (Lammer et al. 2005). During the lifetime of a star the HZ may also be shifted towards greater distances. According to computations by von Bloh et al. (2005), the Earth will no longer be in the HZ after 500 million years. The F-type stars are more massive and hotter than our Sun, which causes a

\footnotetext{
5 The time scale for synchronous rotation depends on the orbital radius just as Goldreich \& Soter (1966) found.
} 

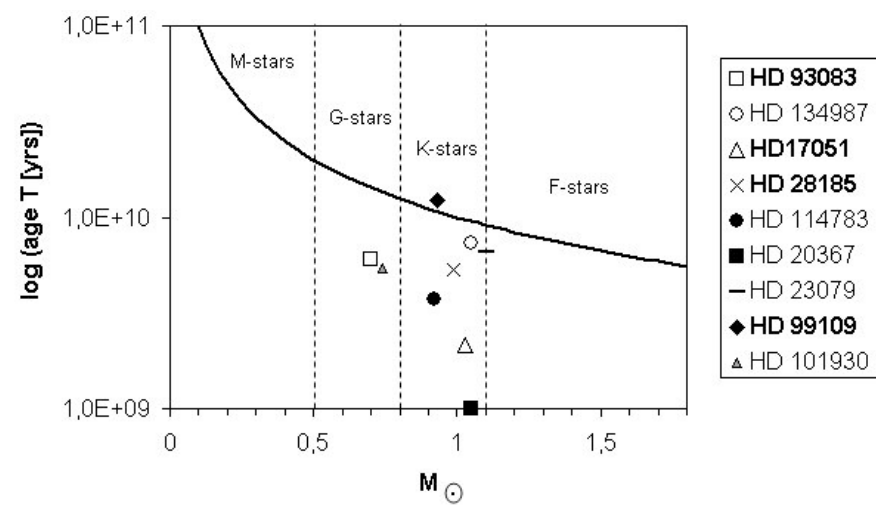

Fig. 6. Lifetime of main sequence stars for different masses (0.05-2 solar masses). The current ages of the stars are given on Jean Schneider's homepage http://www. exoplanets. eu and Saffe et al. (2006).

higher radiation flux, contributing to higher UV radiation (less significant for M-type and K-type stars). This UV radiation is very important for the photochemistry on Earth. The resulting biological activity - represented by bio-signatures or biomarkers in the planet's atmosphere, see Kaltenegger \& Selsis (2007) should be visible in the spectrum of an Earth-like planet. We can conclude that the most promising star types for habitability are the K, G, and F-type stars. However, from an observational point of few, we know that $\mathrm{G}$ and K-type stars are much more numerous than F-type stars. The F-type represents 3.1\%, G-type 8\%, and K-type $13 \%$ of all main sequence stars. The most prominent stars in the Solar environment are the M-type stars, which we excluded from our studies of Trojan planets, because the closeness of the HZ to the central star inhibits the development of a stable atmosphere.

We examined the investigated systems with respect to the spectral type (see Table 2), which is important for the habitability of the planet. Table 2 shows a list of the investigated EPSs with their present age (see Saffe et al. 2006 and Jean Schneider's homepage http://www . exoplanets.eu) and the lifetime of the central star calculated with formula (1). The list is sorted by the increasing mass of the central star. For the examination of the influence on the habitability of a planet through the spectral type of the investigated systems, we calculated the different lifetimes of the stars (see Table 2). We call that a star will leave the main sequence of the HRD when it exhausts its hydrogen and will develop in to a giant star. The time $T$ when the star leaves the main sequence is determined by the formula given in the book by Lang (1980):

$T=1.1 \times 10^{10} \frac{M}{L}$ years.

With the mass-luminosity-relation $L \sim M^{3}$, we get the approximation

$T \approx 10^{10}\left(\frac{M_{\odot}}{M}\right)^{2} \approx 10^{10}\left(\frac{L_{\odot}}{L}\right)^{2 / 3}$,

where mass $M$ of the star and the absolute luminosity $L$ are given in solar units. This respective values are given in Table 2 . In Fig. 6 we plotted age $T$ as function of the mass. One can see that low-mass stars burn their "fuel" more slowly than high-mass stars.

Today the search for extraterrestrial life concentrates on main sequence stars. We have to exclude the systems HD 27442 and HD 188015 from our habitability investigation, because
Table 2. List of the investigated EPSs with their present age (see Saffe et al. 2006 and Jean Schneider's homepage http://www.exoplanets.eu) and the lifetime of the central star calculated with formula (1). The list is sorted by the increasing mass of the central star. Note: All EPSs where the GG moves fully in the $\mathrm{HZ}$ are written in bold.

\begin{tabular}{lllll}
\hline \hline Name & Spec. & $\begin{array}{l}\text { Mass } \\
{\left[M_{\odot}\right]}\end{array}$ & $\begin{array}{l}\text { Present } \\
\text { age } \\
{[\mathrm{Gyrs}]}\end{array}$ & $\begin{array}{l}\text { Lifetime } \\
\text { of the } \\
\text { star [Gyrs] }\end{array}$ \\
\hline HD 93083 & K3V & 0.70 & 6.00 & 20.41 \\
HD 101930 & K1V & 0.74 & 5.39 & 20.00 \\
HD 114783 & K0 & 0.92 & 3.7 & 11.82 \\
HD 99109 & K0 & 0.93 & $\geq 12.20$ & 11.56 \\
HD 28185 & G5 & 0.99 & 5.36 & 10.20 \\
Sun & G2V & 1.00 & 4.5 & 10.00 \\
HD 17051 & G0V & 1.03 & 2.15 & 9.43 \\
HD 134987 & G5V & 1.05 & 7.32 & 9.07 \\
HD 20367 & G0 & 1.05 & 0.87 & 9.07 \\
HD 23079 & (F8)/G0V & 1.10 & 6.53 & 8.27 \\
HD 221287 & F7V & 1.25 & $?$ & 8.00 \\
\hline
\end{tabular}

they are subgiants (see Table 2). We used the work of Saffe et al. (2006) for the approximated present age of the stars given in Table 2 and put them in Fig. 6, to see if the values are above the curve of the estimated lifetime $\mathrm{T}$ of the star. It turns out that HD 99109 is above this curve and consequently too old to hoste a habitable TP. HD 134987 is close to the limit, while the present age for the system HD 221287 is not known.

\subsection{The influence of tidal locking}

The influence of tidal locking could cause the atmosphere of the TP to become unstable. This effect mainly occurs for M-type stars, but also for K-type stars where the HZ is very close to the central star. We have one case in our investigation where this effect appear (HD 101930). We know from the observational data that the GG is only 0.3 AU from the central star (see Table 1). By using the work of Seager \& Hui (2002), we made an approximation for a possible habitable Trojan planet. For synchronous rotation (by using the values of a TP), we get a time scale of $\approx 10^{9}$ yrs. There is a certain probability that, during this time scale, at least primitive life can be formed in this system.

\subsection{Summarising results}

In the case of HD 101930, we have to consider the effect of tidal locking, because the GG is only 0.3 AU from the central star. The spectral types of HD 114783, HD 20367, HD 23079, HD 99109 , and HD 221287 are still not clear. However, the spectra of HD 93083, HD 134987, and HD 17051 are such that the conditions for habitability are good. We can summarise that 3 of the 7 investigated systems that are fully in the HZ (see Table 1) have good conditions for habitability, namely HD 93083, HD 28185 , and HD 17051.

\section{Conclusions}

In their attempt to find life in the universe besides our own, astronomers look for other planetary systems around stars, primarily in the neighbourhood of the Solar System up to several tenths of parsecs. That there are very different orbits for most of the giant planets, found in these systems leads to difficult questions 
about the formation of such systems and also the orbital stability of the planets there. But even more interesting is investigating the possibility of stable terrestrial planets in these systems on low eccentric orbits in the habitable zones around a host star.

In this article we concentrated on possible terrestrial Trojan planets that may stay around the equilateral equilibrium points of a system consisting of a host star and a gas giant. We investigated 12 different EPSs in detail and found that stable regions around these points would allow planets to move there and to stay there for long times on orbits of low eccentricity. We can conclude that 7 of the 12 real systems have good initial conditions for habitability; but in the context of the spectral type of the central star, we find that HD 93083, HD 28185, and HD 17051 have even better conditions for habitability of a possible Trojan planet.

In addition, we compiled a new catalogue of such stable regions for different mass ratios of the primary bodies (host star and GG) and different eccentricities of their orbits. We also determined the stable area by using the results of integrations for $10^{7}$ periods. An integration time of $0.1 \%\left(10^{4}\right.$ periods $)$ already gave a good first approximation, but it turned out that the stability region may be overestimated by up to $20 \%$ (Fig. 4). Therefore we extended the computation time significantly compared to the work of Érdi \& Sandor (2005) and Érdi et al. (2007), by a factor of $10^{4}$.

Special attention has been given to mass ratios in the range of the giants found up to now in EPSs and also to the low eccentricities of their orbits. The results can be used for any EPSs found in the future to tell the probability of having a large enough region around the $L_{4}$ or $L_{5}$ points to host terrestrial planets. This new catalogue is especially useful for single planetary systems when estimating the size of the stable regions around the equilateral Lagrangian points. We emphasise that this catalogue is a useful tool, because the stable regions can be derived be a simple scaling using our results (see Sect. 4). It may also be important for future detection of real Trojan planets in EPS via sophisticated observation techniques from the ground or space.

Acknowledgements. For the realisation of this study we need to thank the Austrian Science Foundation (FWF, project P16024-N05) and the ISSI Institute in Bern, which supported us in the framework of the ISSI team "Evolution of habitable planets". RS did this work in the framework of a Schrödinger grant of the FWF (J2619-N16); Thanks also go to the "Wissenschaftlich-technische Zusammenarbeit Österreich-Ungarn" project A12-2004: Dynamics of extrasolar planetary Systems.

\section{References}

Beaugé, C., Sándor, Zs., Érdi, B., \& Süli, Á. 2007, A\&A, 463, 359

Dvorak, R., \& Schwarz, R. 2005, in A Comparison of the Dynamical Evolution of Planetary Systems, ed. R. Dvorak, \& S. Ferraz-Mello (Springer), 19

Dvorak, R., Pilat-Lohinger, E., Schwarz, R., \& Freistetter, F. 2004, A\&A, 426, 37

Efthymiopoulos, C., \& Sándor, Z. 2005, MNRAS, 364, 253

Érdi, B., \& Sándor, Z. 2005, CeMDA, 92, 113

Érdi, B., Fröhlich, G., Nagy, I., \& Sándor, Zs. 2007a, Proc. of the 4th Austrian Hungarian Workshop on celestial mechanics, ed. Á. Süli, F. Freistetter, \& A. Pál, 85

Érdi, B., Nagy, I., Fróhlich, G., Sándor, Zs., \& Súli, Á. 2007b, MNRAS, submitted

Froeschlé, Cl. 1984, CeMDA, 34, 95

Giorgilli, A., \& Skokos, Ch. 1997, A\&A, 317, 254

Hanslmeier, A., \& Dvorak, R. 1984, A\&A, 132, 203

Holman, M. J., \& Wisdom, J. 1993, AJ, 105, 1987

Jones, B. W., \& Sleep, P. N. 2002, A\&A, 393, 1015

Kasting, J. F., Whitmire, D. P., \& Reynolds, R. T. 1993, Icarus, 101, 108

Kaltenegger, L., \& Selsis, F. 2007, in Extrasolar Planets: Formation, Detection and Dynamics, ed. R. Dvorak (New York: Wiley), in press

Lammer, H., Kulikov, Yu., Penz, T., et al. 2005, in A Comparison of the Dynamical Evolution of Planetary Systems, ed. R. Dvorak, \& S. Ferraz-Mello (Springer), 273

Lang, K. R. 1980, Astrophysical Formulae (Springer), 511

Laughlin, G., \& Chambers, J. E. 2002, AJ, 124, 592

Lohinger, E., \& Dvorak, R. 1993, A\&A, 280, 683

Lohinger, E., Froeschlé, C., \& Dvorak, R. 1993, CeMDA, 56, 315

Lichtenegger, H. 1984, CeMDA, 34, 357

Marchal, C. 1990, The three-Body Problem (Elsevier), 49

Marzari, F., \& Scholl, H. 1998, A\&A, 339, 278

Menou, K., \& Tabachnik, S. 2003, AJ, 583, 473

Morbidelli, A., Levison, H. F., Tsiganis, K., \& Gomes, R. 2005, Nature, 435, 462 Nauenberg, M. 2002, AJ, 124, 2332

Pál, A., \& Sándor, Zs. 2003, Proc. of the 3rd Austrian-Hungarian Workshop on Trojans and related topics, ed. F. Freistetter, R. Dvorak, \& B. Érdi, 25

Rivera, E. J., Lissauer, J. J., R. P., Marcy, G. W., et al. 2005, AAS, 37, 1487

Skokos, Ch., \& Dokoumetzidis, A. 2001, A\&A, 367, 729

Saffe, C., Gómez, M., \& Chavero, C. 2006, A\&A, 443, 609

Schwarz, R. 2005, Ph.D. Thesis, University of Vienna, online database: http://media.obvsg.at/dissdb

Schwarz, R. 2007, Proc. of the 4th Austrian Hungarian Workshop on celestial mechanics, ed. Á. Süli, F. Freistetter, \& A. Pál, 149

Schwarz, R., Pilat-Lohinger, E., Dvorak, R., Érdi, B., \& Sándor, Zs. 2005, Astrobiology, 5, 579

Schwarz, R., Dvorak, R., Pilat Lohinger, E., Süli, Á., \& Érdi, B. 2007a, A\&A, 462,1165

Schwarz, R., Dvorak, R., Süli, Á., \& Érdi, B. 2007b, AN, submitted

Seager, S., \& Hui, L. 2002, ApJ, 574, 1004

Tuckness, D. G. 1995, CeMDA, 61, 1

von Bloh, W., Bounama, C., \& Franck, S. 2003, in A Comparison of the Dynamical Evolution of Planetary Systems, ed. R. Dvorak, \& S. Ferraz-Mello (Springer), 287

Udry, S., Bonfils, X., Delfosse, X., et al. 2007, A\&A, 469, 43 